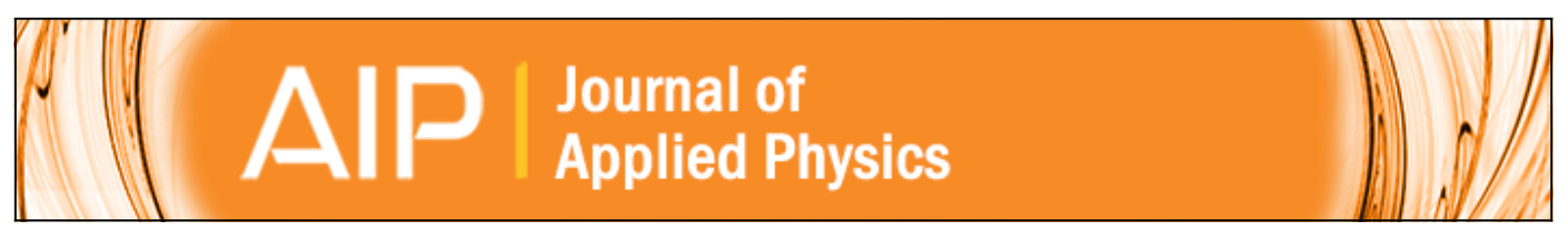

\title{
Fatigue effect in ferroelectric crystals: Growth of the frozen domains
}

V. Ya. Shur, A. R. Akhmatkhanov, and I. S. Baturin

Citation: Journal of Applied Physics 111, 124111 (2012); doi: 10.1063/1.4729834

View online: http://dx.doi.org/10.1063/1.4729834

View Table of Contents: http://scitation.aip.org/content/aip/journal/jap/111/12?ver=pdfcov

Published by the AIP Publishing

\section{Articles you may be interested in}

Formation of dendrite domain structures in stoichiometric lithium niobate at elevated temperatures

J. Appl. Phys. 112, 104113 (2012); 10.1063/1.4766308

Polarization reversal and jump-like domain wall motion in stoichiometric LiTaO3 produced by vapor transport equilibration

J. Appl. Phys. 111, 014101 (2012); 10.1063/1.3673601

Ferroelectric domain structure of PbZr0.35Ti0.65O3 single crystals by piezoresponse force microscopy

J. Appl. Phys. 110, 052003 (2011); 10.1063/1.3623768

Domain reversal and nonstoichiometry in lithium tantalate

J. Appl. Phys. 90, 2949 (2001); 10.1063/1.1389525

Domain reversal and wall structure of $180^{\circ}$ ferroelectric domains in $\mathrm{LiTaO} 3$ crystals

AIP Conf. Proc. 535, 183 (2000); 10.1063/1.1324455

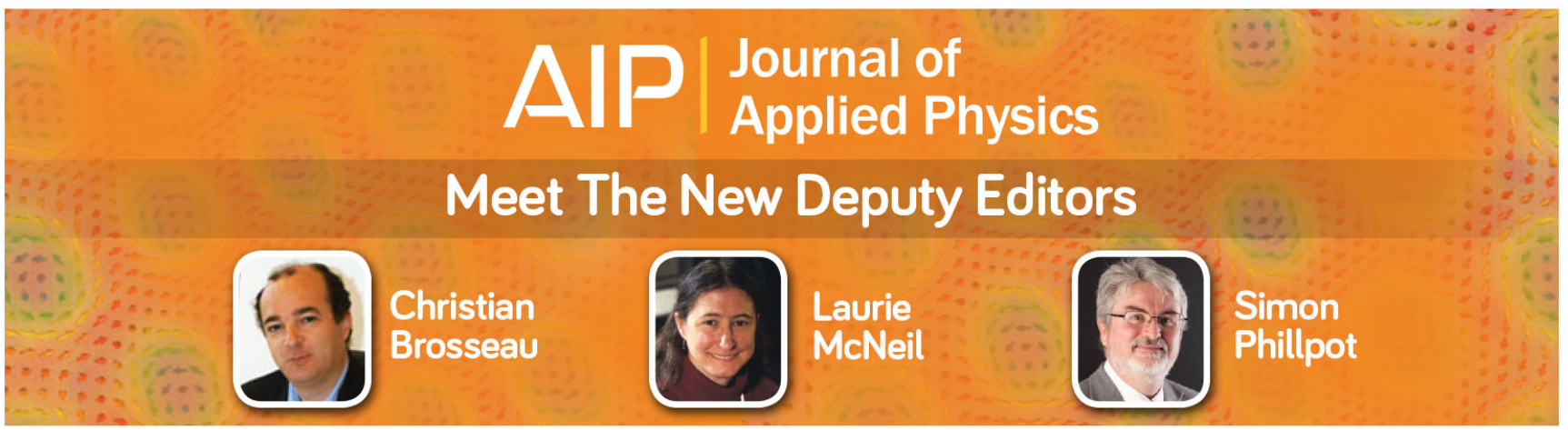




\title{
Fatigue effect in ferroelectric crystals: Growth of the frozen domains
}

\author{
V. Ya. Shur, ${ }^{\text {a) }}$ A. R. Akhmatkhanov, and I. S. Baturin \\ Ferroelectric Laboratory, Institute of Natural Sciences, Ural Federal University, 620000 Ekaterinburg, \\ Russia and Labfer Ltd, 620014 Ekaterinburg, Russia
}

(Received 11 March 2012; accepted 19 May 2012; published online 22 June 2012)

\begin{abstract}
The model of the fatigue effect during cyclic switching caused by growth of the frozen domain area with charged domain walls has been proposed. It was claimed on the basis of the previous experimental results that for switching in increasing field the frozen domain area started to grow at the given sub-threshold field value and stopped at the threshold field. The influence of the shape and frequency of the field pulses used for cyclic switching has been considered. The uniaxial ferroelectric stoichiometric lithium tantalate single crystals produced by vapor transport equilibration with record low value of coercive field have been chosen as a model material for experimental verification of the model. The formation of the charged domain walls as a result of cyclic switching has been revealed by analysis of the domain images obtained by optical and Raman confocal microscopy. It has been shown that the fatigue degree is equal to the fraction of the frozen domain area. The experimental dependence of the switched charge on the cycle number has been successfully fitted by modified Kolmogorov-Avrami formula. The experimentally observed frequency independence of fatigue profile for rectangular pulses and frequency dependence for triangular pulses has been explained by proposed model. (C) 2012 American Institute of Physics. [http://dx.doi.org/10.1063/1.4729834]
\end{abstract}

\section{INTRODUCTION}

The effect of polarization fatigue (decrease of the residual polarization during cyclic switching) being one of the major problems in FeRAM attracted much attention of ferroelectric society. ${ }^{1}$ The most part of the published experimental data is related to study of the fatigue in PZT $\left(\mathrm{PbZr}_{\mathrm{x}} \mathrm{Ti}_{1-\mathrm{x}} \mathrm{O}_{3}\right)$ thin films. ${ }^{2}$ However, the nature of this effect is still under discussion.

The main experimental characteristic of fatigue process is so called fatigue profile representing the dependence of the residual polarization on the number of the switching cycles $\mathrm{P}_{\mathrm{r}}(\mathrm{N})$. The main fatigue mechanisms and models developed for explanation of the fatigue effect were reviewed earlier., ${ }^{2,3}$

The passive layer model suggests that the cyclic switching of the sample results in growth of the low-dielectric constant interface layer. ${ }^{4-6}$ As a result, the field applied to the bulk of ferroelectric decreases which leads to decrease of the residual polarization.

According to the wall pinning mechanism, the immobilization of domain walls caused by their interaction with defects results in suppression of the switching ability of ferroelectric domains. ${ }^{7}$ The pinning of the domain walls is considered to be the result of interaction between the bound charges on the domain walls and the mobile carriers.

In the seed inhibition mechanism, the switching ability of seeds is suppressed in the embryonic state before they can create a macroscopic domain. ${ }^{8}$ Fatigue is associated with blocking seeds by ionic or electronic defects accumulated near the interface electrode-ferroelectric. The widely accepted candidates for mobile carriers necessary for realiza-

\footnotetext{
a) Author to whom correspondence should be addressed. Electronic mail: vladimir.shur@usu.ru.
}

tion of wall pinning and seed inhibition mechanisms are oxygen vacancies and/or electrons and holes injected from the electrodes to the ferroelectric bulk through the surface dielectric layer.

According to kinetic approach, the fatigue phenomenon is a result of self-organized domain kinetics due to retardation of bulk screening of depolarization field..$^{9-11}$ The formation of spatially nonuniform internal bias field during cycling (kinetic imprint effect) slows the domain kinetics in some regions leading to formation of the kinetically frozen domains.

The model of charged ionic defects migration considers the preferential electromigration of ionic defects (for example, oxygen vacancies) driven by ac field to the regions near the electrode-ferroelectric interface. ${ }^{12,13}$ The defects entrapment eventually leads to the loss of the residual polarization. Two alternative functions for fitting of the fatigue profiles in PZT thin films were obtained using this model ${ }^{13-15}$

$$
P_{r}(N)=P_{r 0}(a N+1)^{-m},
$$

where $P_{r 0}$ is the initial value of residual polarization, a is the constant, $\mathrm{m}$ is the decay constant.

And

$$
P_{r}(N)=P_{r 1} \exp (-b N)+P_{r 2},
$$

where $P_{r 1}+P_{r 2}=P_{r 0}, b$ is the constant.

The empirical fit of fatigue profile by stretched exponent was also used. ${ }^{16}$

Only small part of the publications contains the information about reconstruction of the domain structure during fatigue cycling. It was shown by direct observations using scanning probe microscope that fatigue in PZT thin films manifested itself through arising and growth of complicated 
structure of non-switchable regions ("frozen domains"). 17,18 Similar effect was revealed in $\mathrm{BaTiO}_{3}$ single crystals during cyclic switching with Ag electrodes. ${ }^{19,20}$ It was shown that the domain structure of fatigued samples represented "aggregate of antiparallel domains-wedges" (needle-like domains) which did not penetrate the entire thickness of the crystal, but ended somewhere in the bulk. ${ }^{19,20}$ Thus, the formation of the stable domain structure with charged walls as a result of cyclic switching was revealed.

\section{A. Growth of the frozen domain area in constant field}

The formation and evolution of the domain structure with stable charged domain walls (CDW) were studied in congruent lithium niobate $\mathrm{LiNbO}_{3}(\mathrm{CLN}){ }^{21}$ The mechanism of self-maintained propagation of CDW governed by selfconsistent electrostatic interaction between the steps of the wall was proposed. ${ }^{21}$ It was shown that during switching under application of rectangular field pulse ("constant field") using transparent solid-state ITO electrodes the domains nucleated at polar surface grew in forward direction and coalesced at $\mathrm{Z}+$ but did not reach $\mathrm{Z}$ - surface thus forming large isolated domains bounded by $\mathrm{CDW}$ (Fig. 1). The created CDW represented the frozen domain area, whereas subsequent application of the reversed fields did not decrease its area. $^{21}$ The growth of frozen domains with CDW was governed by sideways motion of the CDW boundary characterized by velocity $v_{C D W}$.

It was demonstrated that the CDW growth started at the field value $E_{t h}{ }^{C D W}=17 \mathrm{kV} / \mathrm{mm}$, which was essentially lower than the threshold field for switching from single domain state $\left(E_{t h}=21 \mathrm{kV} / \mathrm{mm}\right){ }^{21}$ Thus, the CDW formation and growth were obtained in sub-threshold fields ranged from 17 to $21 \mathrm{kV} / \mathrm{mm}$. It was observed experimentally that the CDW growth was changed by classical growth of through domains with neutral domain walls in fields above $21 \mathrm{kV} / \mathrm{mm}^{21}$

\section{B. Application of Kolmogorov-Avrami formula}

It was shown that the evolution of ferroelectric domain structure during polarization reversal in electric field could be considered as an analog of the first order phase transformation process. ${ }^{22-24}$ In this case, the local electric field was

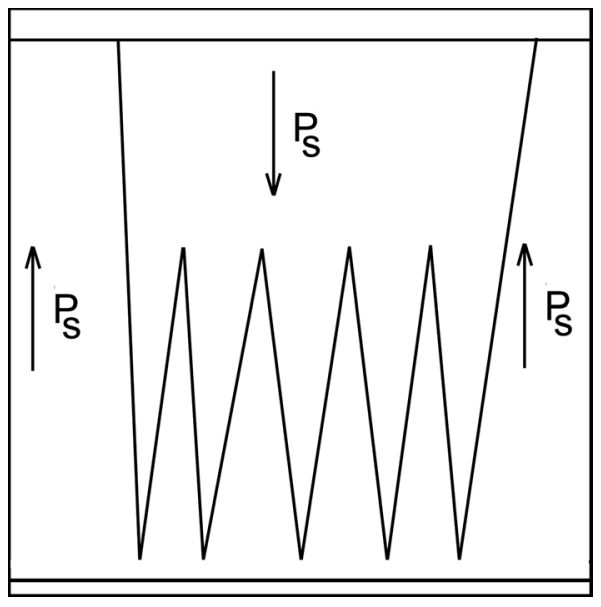

FIG. 1. The scheme of the domain with CDW. the driving force of the process. The Kolmogorov-Avrami (K-A) formula ${ }^{25,26}$ modified for the finite media was used successfully for quantitative description of the dependence of switched charge and switching current on time during polarization reversal. ${ }^{27-29}$ In this paper, K-A formula has been applied for characterization of the frozen domain growth.

The K-A model considers the phase transformation in infinite media under the assumption that the mean size of the individual transformed regions is small as compared to the sample area. It is formulated for the process with the great number of nuclei randomly distributed over the volume and time. Assuming spatially uniform nucleation probability and the equal value of growth velocity for all growing domains at a given moment, the following expression for the time dependence of relative fraction of non-switched volume $q(t)$ is obtained:

$$
q(t)=\exp \left[-\int_{0}^{t} \alpha(\xi) \mathrm{V}(R(\xi, t)) d \xi\right]
$$

where $\alpha$ is the nucleation probability per volume, $\xi$ is the arising time of the nucleus, $V(R)=c R^{n}$ is the volume occupied by individual domain at the moment $t, c$ is the shape constant, $R(\xi, t)$ is the domain radius, $n$ is the dimensionality of the problem, which takes on the integer values only.

It should be noted that the dimensionality of the domain growth in finite media can differ from the space dimensionality and depends on the domain shape: $n$ is equal to 3 for the growth in the bulk, $n=2$ for cylinder domains, and $n=1$ for lamella domains. ${ }^{22,29}$

Two limiting situations of the nucleation process were considered in the K-A model. Following Kolmogorov ${ }^{25}$ classification: the first is $\alpha$ model, when the nuclei (new domains) appear through the whole switching process with probability $\alpha(t)$ per unit volume, and the second is $\beta$ model when all nuclei appear at the very beginning of the process with the density $\beta$ per unit volume. The modification of the $\mathrm{K}-\mathrm{A}$ formula for the finite media, which takes into account the change of the shape constant, when the growing domains touch the boundary of the switched volume, allowed to derive the following formulas for constant value of the switching field during the whole process: ${ }^{29}$

for $\alpha$ model,

$$
\begin{aligned}
q(t) & =\exp \left[-(n+1)^{-1} c \alpha v^{n} t^{n+1}\left(1-t / t_{\mathrm{m}}\right)\right] \\
& =\exp \left[-\left(t / t_{0 \alpha}\right)^{n+1}\left(1-t / t_{\mathrm{m}}\right)\right]
\end{aligned}
$$

for $\beta$ model,

$q(t)=\exp \left[-c \beta v^{n} t^{n}\left(1-t / t_{\mathrm{m}}\right)\right]=\exp \left[-\left(t / t_{0 \beta}\right)^{n}\left(1-t / t_{\mathrm{m}}\right)\right]$,

where $v$ is the domain wall velocity, $t_{m}$ is the time constant which accounts for the touch of the growing domains with the boundary of the switching volume, $t_{0 \alpha}=\left[(n+1)^{-1} c \alpha v^{n}\right]^{-1 /(n+1)}$ and $t_{0 \beta}=\left(c \beta v^{n}\right)^{-1 / n}$ are the characteristic times of the process. 
Equations (4) and (5) can be used for $t<t_{m}$ only. Moreover, the proposed consideration is invalid, when all domains have touched the boundary $t=t_{c r}<t_{m}{ }^{29}$

For anisotropic case, when the critical times $\left(t_{c r}\right)_{i}$ differ essentially for various growth directions $i$ and the minimal critical time is much shorter than the total switching time, the whole process can be divided into stages with different growth dimensionality. The stepwise decrease of the problem dimensionality ( $3 \mathrm{D} \rightarrow 2 \mathrm{D}$ or $2 \mathrm{D} \rightarrow 1 \mathrm{D}$ ) is obtained at the time of catastrophe $t_{c a t}=\min \left(t_{c r}\right)_{i}$. Thus, the final stage of the switching process is described by the formula (5) with the reduced integer value of growth dimensionality $n \rightarrow(n-1)$ and negligible effect of domain interaction with boundaries. ${ }^{29}$

For $\beta$ model,

$$
\begin{gathered}
q(t)=\exp \left[-\left(t / t_{01}\right)^{n}\left(1-t / t_{\mathrm{m}}\right)\right], \quad \text { for } \quad t \leq t_{\text {cat }}, \\
q(t)=\exp \left[-\left(t / t_{02}\right)^{n-1}\right], \quad \text { for } \quad t \geq t_{\text {cat }},
\end{gathered}
$$

where $t_{01}$ and $t_{02}$ are the characteristic times of the process before and after $t_{c a t}$, respectively.

Thus, it was known that cyclic switching leaded to formation of the frozen domain areas with charged domain walls. The growth of the frozen domain area could be described using K-A formula modified for switching in finite-size media.

\section{MODEL OF THE FATIGUE PROCESS CAUSED BY GROWTH OF THE FROZEN DOMAINS}

On the basis of the experimental results obtained earlier, we propose to describe the fatigue process as the formation and growth of the frozen domain area with CDW. It is necessary to take into account that forward polarization reversal ("direct switching") during cyclic switching by triangular pulses occur in increasing field. Moreover, the switching in increasing field is obtained for rectangular pulses during the ramp time (Fig. 2(b)). The growth of the frozen domain area in increasing field occurred in experimentally revealed field range from $E_{t h}{ }^{C D W}$ to $E_{t h}$ ("sub-threshold field limits for CDW formation and growth"). ${ }^{21}$ Whereas at the threshold field, the domain kinetics changed qualitatively from CDW growth to the classical growth of domains with neutral domain walls. ${ }^{21}$
The dependence of sideways motion velocity of the CDW boundary on external field $E_{e x}$ can be approximated by the following formula:

$$
v_{C D W}\left(E_{e x}\right)=\mu\left(E_{e x}-E_{t h}{ }^{C D W}\right), \quad \text { for } \quad E_{e x}<E_{t h},
$$

where $\mu$ is the mobility of CDW boundary.

CDW formation typically starts at the needle-like residual domains which appear in any real ferroelectric during cyclic switching. At sub-threshold fields, the residual domains demonstrate the lateral growth on $\mathrm{Z}+$ polar surface by step generation followed by "forward" growth (step growth in polar direction). ${ }^{30}$ In the field range $E_{t h}{ }^{C D W}<E_{e x}<E_{t h}$, the tips of the needle-like domains do not reach the opposite polar surface due to termination of the step growth. ${ }^{21,31}$ The electrostatic interaction between charged ends of the steps leads to the slowing and termination of the approaching neighboring step ends, thus preventing step annihilation. The spreading of the step termination process leads to increase of the step concentration (the bound charge at the wall) and formation of a CDW fragment abnormally deflected from the polar direction. ${ }^{21}$ The high value of depolarization field at that domain wall fragment leads to generation of the additional spikes. The spikes tips propagate to $\mathrm{Z}-$ and stop at the gap. This cyclic process occurs over and over, thus providing formation of the quasi-regular dented structure by sideways motion of the CDW boundary.

Let us consider the cyclic switching by application of bipolar pulses with amplitude $E_{\max }$ above $E_{t h}$, which is enough for the complete switching. In this case CDW growth is obtained during each cycle only in the time interval $\Delta t_{C D W}$, when $E_{t h}{ }^{C D W}<E_{e x}(t)<E_{t h}$, thus leading to "discrete growth" of the total frozen domain area

$$
\Delta t_{C D W}=\Delta E_{C D W} / R
$$

where $R=\mathrm{d} E_{e x} / \mathrm{d} t$ is rate of field rise.

For cyclic switching by bipolar triangular pulses (Fig. 2(a)),

$$
\Delta t_{C D W}=\Delta E_{C D W} /\left(4 E_{\max } f_{s w}\right),
$$

where $\Delta E_{C D W}=E_{t h}-E_{t h}{ }^{C D W}, E_{\max }$ is a field pulse amplitude, $f_{s w}$ is a field pulse frequency.

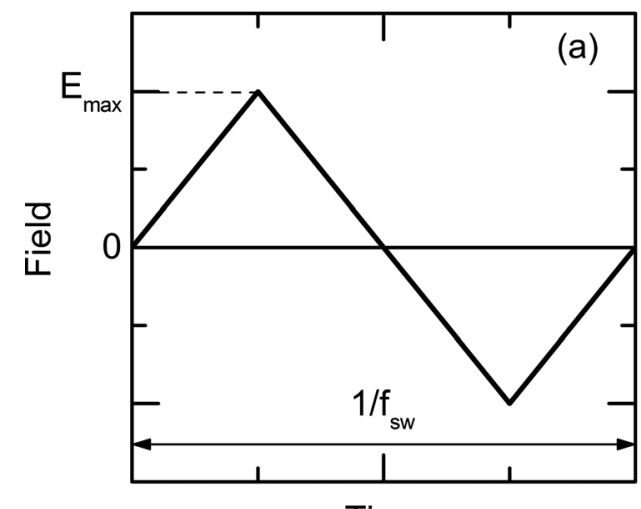

Time

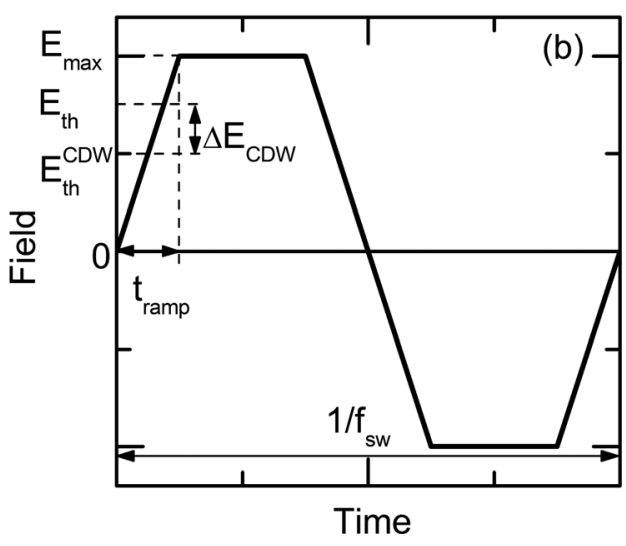

FIG. 2. The time dependence of the applied field for bipolar (a) bipolar triangular and (b) rectangular pulses. 
For cyclic switching by bipolar rectangular pulses (Fig. 2(b)),

$$
\Delta t_{C D W}=t_{\text {ramp }} \Delta E_{C D W} / E_{\max },
$$

where $t_{\text {ramp }}$ is ramp time.

$\mathrm{K}-\mathrm{A}$ approach can be used for the discrete process also with change of the process time $t$ by $N \Delta t_{C D W}$. In this case for $\beta$ model, the dependence of the relative switching charge on the number of switching cycles can be expressed by

$$
q(N)=\exp \left[-\left(N \Delta t_{C D W} / t_{0 b}\right)^{n}\left(1-N \Delta t_{C D W} / t_{\mathrm{m}}\right)\right] .
$$

This expression can be rewritten as

$$
q(N)=\exp \left[-\left(N / N_{0 \beta}\right)^{n}\left(1-N / N_{\mathrm{m}}\right)\right]
$$

where $N_{0 \beta}=t_{0 \beta} / \Delta t_{C D W}, N_{\mathrm{m}}=t_{\mathrm{m}} / \Delta t_{C D W}$.

The value $N_{0 \beta}$ can be considered as the characteristic rate of the fatigue process. The obtained Eq. (13) is the ana$\log$ of K-A formula for fatigue during cyclic switching. The parameters of the fatigue process $\left(N_{O \beta}\right.$ and $\left.N_{\mathrm{m}}\right)$ can be obtained from approximation of the experimental data. It is seen from Eqs. (10), (11), and (13) that the $N_{O \beta}$ is frequency dependent for triangular pulses and frequency independent for rectangular pulses. It is necessary to point out that our model can be applied for sufficient field amplitude of the switching pulses $\left(E_{\max }>E_{t h}\right)$. This relation is the necessary condition for complete switching at the beginning of the fatigue cycling. The violation of this important condition of fatigue cycling leads to erroneous experimental results and hampers their interpretation.

The experimental study of the domain structure evolution during cyclic switching and its correlation with the shape of the $P_{r}(N)$ fatigue profile is still missing nowadays. ${ }^{2}$ The main part of experimental study of fatigue kinetics was realized in thin ferroelectric films which hampered the direct observation of the domain kinetics. This was the reason to study the fatigue process in model ferroelectric single crystals with simple domain structure, which could be visualized nondestructively by several methods. ${ }^{32}$

The role of the "frozen domains" in fatigue effect was studied in the model ferroelectric crystal, which has been chosen according to the following requirements: (1) uniaxial ferroelectric with simple domain structure and $180^{\circ}$ domain walls only, (2) high crystal quality, (3) domain structure easily reversible in low electric field, (4) high enough transition temperature, (5) ability to use the nondestructive visualization of the domain structure in the bulk with sub-micron spatial resolution by Raman confocal microscopy (RCM).

The detail analysis of the experimental results demonstrates that lithium tantalate single crystal produced by vapor transport equilibration (VTE-LT) is the best candidate for such investigation. ${ }^{33-35}$ It belongs to the family of lithium niobate (LN) and lithium tantalate (LT) crystals, which are the most popular nonlinear-optical ferroelectric materials. ${ }^{36}$ This uniaxial ferroelectric demonstrates the lowest coercive field within all representatives of LN and LT family (about 200 times lower than in CLN). ${ }^{33}$ VTE-LT possesses almost ideal composition with low concentration of the bulk non- stoichiometry defects achieved by long-time annealing at the temperature above $1000{ }^{\circ} \mathrm{C}$ in the Li-rich atmosphere. ${ }^{37}$ It is necessary to point out that the domain walls in all crystals of LN and LT family can be visualized in the bulk using RCM $^{38,39}$ The combination of all required properties leads us to choose VTE-LT as the model material for investigation of the experimental verification of the proposed fatigue model.

\section{EXPERIMENT}

The process of fatigue during cyclic switching was investigated in Z-cut 0.8 -mm-thick single-crystalline wafers of VTE-LT (Silicon Light Machines, CA). The typical sample area was about $20 \times 10 \mathrm{~mm}^{2}$. The annealing of congruent lithium tantalate (CLT) wafers with prereacted Li-rich powder of $70 \% \mathrm{LiO}_{2}$ and $30 \% \mathrm{Ta}_{2} \mathrm{O}_{5}$ during $100 \mathrm{~h}$ at $1100^{\circ} \mathrm{C}$ was used during VTE process. ${ }^{34}$

In our experiments, we used two different types of electrodes: (1) liquid electrodes based on saturated aqueous solution of $\mathrm{LiCl}$; (2) solid state metal electrodes (100-nm-thick $\mathrm{Ni}$ ) or conductive transparent oxide (200-nm-thick $\operatorname{In}_{2} \mathrm{O}_{3}: \mathrm{Sn}$ (ITO)) deposited by DC ion plasma sputtering. The circular solid state electrodes (2-3 $\mathrm{mm}$ in diameter) of the same material were deposited on both polar surfaces. All switching experiments were performed at room temperature.

Programmable arbitrary shaped voltage signals were generated by NI PCI-6251 multifunctional DAQ board, amplified by high-voltage amplifier TREK 20/20C and applied to the electrodes. Precision series resistor $(0.2,2,20$, or $200 \mathrm{k} \Omega$ depending on current range) was used for recording of the switching current. The switching charge was obtained by digital integration of the switching current data. Switching area was supposed to be equal to the area of electrode. Triangular bipolar switching field pulses with frequency range from 1 to $100 \mathrm{~Hz}$ and amplitude up to $1500 \mathrm{~V} / \mathrm{mm}$ were used to obtain hysteresis loop during cyclic switching.

The static domain structure on polar surface and $\mathrm{Y}$ cross-sections was visualized after selective chemical etching in concentrated hydrofluoric acid (HF) for about $30 \mathrm{~min}$. The domain images were acquired by Olympus BX51 and Carl Zeiss LMA 10 optical microscopes in transmitted, reflected, phase contrast, and dark field modes.

The Raman confocal microscopy used for domain visualization in the bulk was realized on the basis of Probe

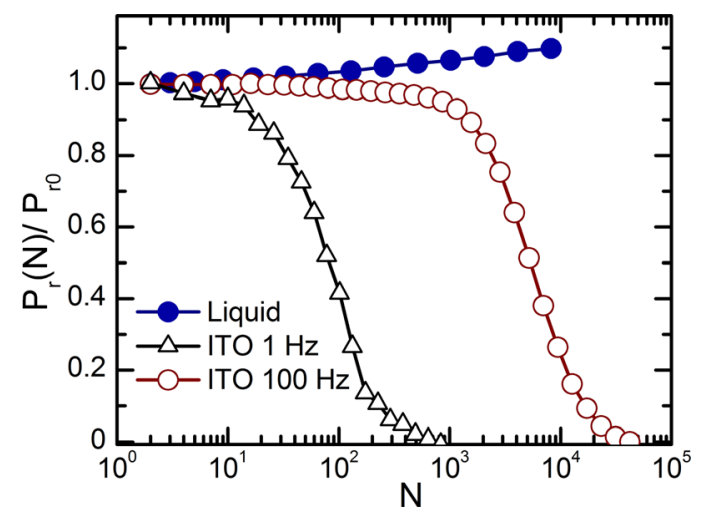

FIG. 3. The dependence of the residual polarization on the number of switching cycles for liquid and ITO electrodes. 


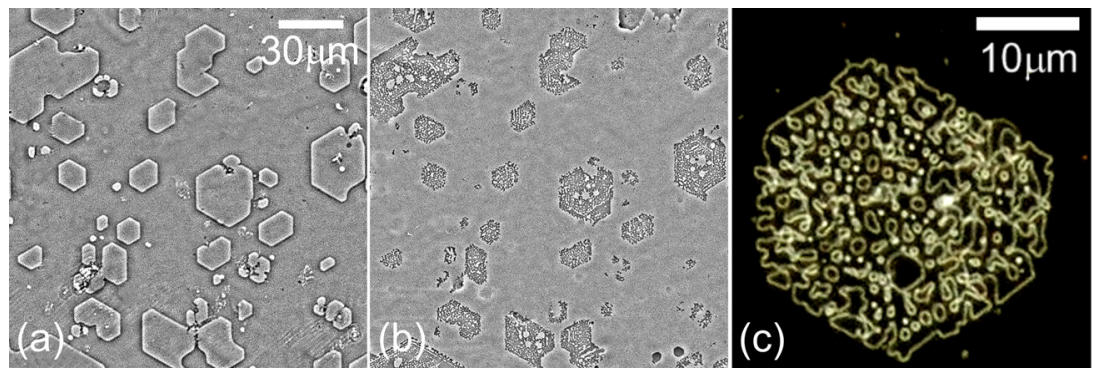

FIG. 4. The domain patterns revealed by etching on (a) $\mathrm{Z}+$, (b) and (c) $\mathrm{Z}$ - polar surfaces after partial fatigue $(\gamma=0.36)$. Visualization by optical microscopy: (a) and (b) phase contrast, (c) dark field.

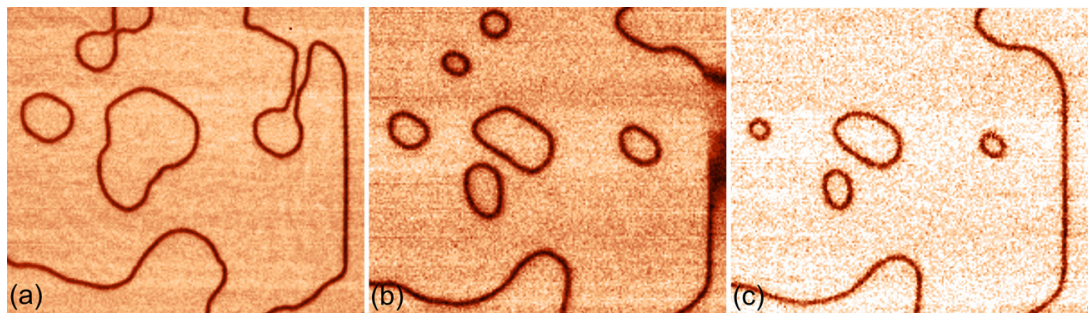

FIG. 5. Domain images revealed by Raman confocal microscopy after partial fatigue at the different depth from Z- surface: (a) $1 \mu \mathrm{m}$, (b) $20 \mu \mathrm{m}$, (c) $45 \mu \mathrm{m}$. Scan size is $50 \mu \mathrm{m}$.

NanoLaboratory NTEGRA Spectra (NT-MDT, Russia) containing high resolution confocal optical microscope and Raman spectrometer. The Raman spectra were recorded in $Z(x x) \bar{Z}$ configuration at room temperature within $2 \mathrm{D}$ scanning with $100 \mathrm{~nm}$ step for obtaining the bulk domain images at the given depth (distance from the polar surface). The diode-pumped solid state laser (Cobolt $\mathrm{AB}$, Sweden) with $\lambda=473 \mathrm{~nm}$ and power $50 \mathrm{~mW}$ was used as a pumping source. Laser focusing was performed by objective $100 \times(\mathrm{NA}=0.95)$ mounted in inverted optical microscope Olympus IX71. The estimated lateral resolution was about $300 \mathrm{~nm}$ and the depth resolution was about $500 \mathrm{~nm}$.

\section{EXPERIMENTAL VERIFICATION OF THE PROPOSED MODEL}

We have demonstrated that the fatigue behavior in VTELT samples strongly depends on the type of electrodes. The samples with liquid electrodes are essentially more resistant to fatigue as compared with the samples with solid electrodes. This fact was revealed earlier in barium titanate crystals. ${ }^{40,41}$

In VTE-LT with liquid electrodes, the fatigue effect is absent after 10000 switching cycles (amplitude $500 \mathrm{~V} / \mathrm{mm}$,

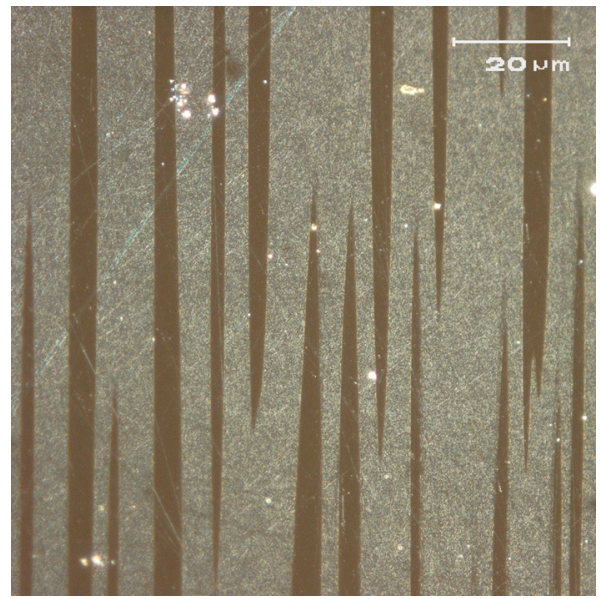

FIG. 6. The domain structure revealed by etching after partial fatigue $(\gamma=0.36)$ on $\mathrm{Y}$ cross section. Optical microscopy. frequency $10 \mathrm{~Hz}$ ). Moreover, the weak wake-up effect (increase of the switching charge by $7 \%$ ) has been observed instead of fatigue (Fig. 3). However, the situation has changed drastically while using solid state electrodes. The same switching test has led to remarkable fatigue effect (the residual polarization has reduced drastically) (Fig. 3). According to this fact, we have chosen the solid electrodes for following study of the fatigue effect.

The fatigue process has been characterized by dependence of the fatigue degree $\gamma$ on the number of switching cycles $N$

$$
\gamma(N)=1-P_{r}(N) / P_{r 0}
$$

It is known that it is quite challenging to visualize the domain structure in VTE-LT by optical microscopy. ${ }^{35}$ Thus, we have used the selective chemical etching of polar surfaces and cross-sections for revealing the domain structure before
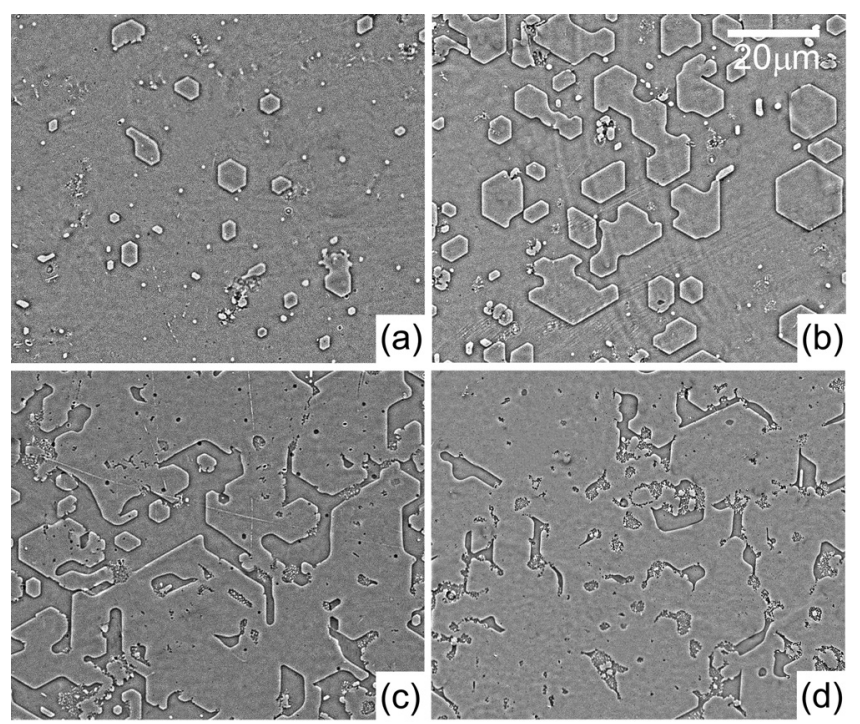

FIG. 7. The frozen domain structures on $\mathrm{Z}+$ polar surface revealed by etching for various values of fatigue degree $\gamma$ : (a) 0.15 , (b) 0.36 , (c) 0.62 , (d) 0.9 . 


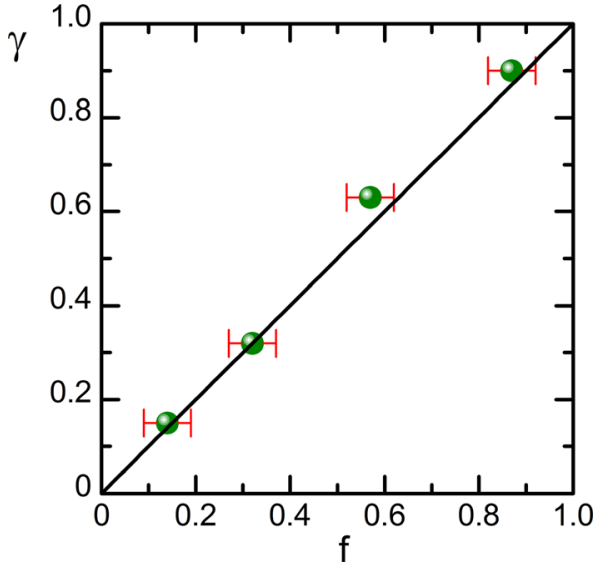

FIG. 8. The dependence of the fatigue degree on the fraction of the frozen domain area.

its optical visualization (Fig. 4). The applied experimental technique allows us to demonstrate that fatigue effect is accompanied by formation and growth of the residual "frozen" (non-switchable) domains.

The domain images obtained after partial fatigue $(\gamma=0.36$ after 1800 switching cycles) on both polar surfaces and $\mathrm{Y}$ cross-section allow us to study the formed frozen domain structure. The usual polygonal domain shapes with walls oriented along Y directions have been obtained on $\mathrm{Z}+$ surface only (Fig. 4(a)). The domain shapes on Z- surface differed qualitatively (Fig. 4(b)). The whole area corresponding to the domain on $\mathrm{Z}+$ surface has been covered by dense irregular shaped domain structure with average period below $2 \mu \mathrm{m}$ (Fig. 4(c)).

The higher resolution images of the domain structure at Z- surface have been obtained by RCM (Fig. 5). ${ }^{39}$ The comparison of the images obtained at different depth from the polar surface allows to reveal the shallow domain structure with charged domain walls. The similar domain images have been obtained for the charged domain walls which appear during polarization reversal in CLN with ITO electrodes. ${ }^{21}$

The needle-like domains with charged walls have been revealed on Y cross-section (Fig. 6). Thus, the obtained experimental results demonstrate that the fatigue effect in VTE-LT with solid state electrodes is accompanied by formation and growth of the frozen domains which represent the areas with CDW (Fig. 1).

We have analyzed the set of domain structure images after various numbers of switching cycles $N$ under the electro-
TABLE I. The values of fatigue process parameters, obtained from approximation of experimental data $q(N)$.

\begin{tabular}{lcc}
\hline \hline & $f_{s w}=1 \mathrm{~Hz}$ \\
$N_{O \beta}(2 \mathrm{D})$ & & $83 \pm 4$ \\
$N_{\mathrm{m}}(2 \mathrm{D})$ & & $250 \pm 30$ \\
$\mathrm{~N}_{0 \beta}(1 \mathrm{D})$ & $98 \pm 4$ \\
& $f_{s w}=100 \mathrm{~Hz}$ & \\
$N_{O \beta}(2 \mathrm{D})$ & & $5070 \pm 80$ \\
$N_{\mathrm{m}}(2 \mathrm{D})$ & $13700 \pm 500$ \\
$\mathrm{~N}_{0 \beta}(1 \mathrm{D})$ & $7100 \pm 100$ \\
\hline \hline
\end{tabular}

des of equal sizes deposited at the neighboring parts of the sample (Fig. 7). The image analysis allows us to extract the fraction of the frozen domain area for given number of switching cycles

$$
f(N)=A_{f d}(N) / A_{e l}
$$

where $A_{f d}(N)$ is the frozen domain area after $N$ switching cycles and $A_{e l}$ is the area of the electrode.

It has been shown that the obtained $f(N)$ values are equal (within the experimental error) to $\gamma(N)$ revealed from the cyclic switching data (Fig. 8). This fact confirms directly that fatigue effect is due to enlarging of CDW area in studied crystals.

The detailed study of the growth of the area covered by frozen domains during cycling has been performed (Fig. 7). It has been revealed from the domain images that the fatigue starts with the formation of isolated hexagonal frozen domains at $\mathrm{Z}+$ surface (Fig. 7(a)). The frozen domains grow, maintaining hexagonal shape during further cyclic switching. The smooth merging of the frozen domain areas occurs (Figs. 7(b)-7(d)). Such behavior qualitatively differs from the jumplike change of the domain shape, ${ }^{42}$ which is typical for the kinetics of the usual domains in VTE-LT. ${ }^{35}$

In terms of the proposed approach, the kinetics of fatigue effect represents the growth and merging of the CDW areas. Thus, the experimental dependence of the fraction of switched charge on the cycle number $q(N)$ can be fitted by K-A formula modified for finite media (13) (Fig. 9). ${ }^{29}$ The obtained results demonstrate predomination of $\beta(2 \mathrm{D})$ process for $q$ ranged from 0.7 to 0.4 . The exponential decrease observed for $q<0.4$ corresponds to $\beta(1 \mathrm{D})$ process (Fig. 8). The obtained values of fitting parameters are presented in Table I. The obtained 2D $\rightarrow 1 \mathrm{D}$ geometrical catastrophe (transition from two-dimensional to one-dimensional growth) takes place,
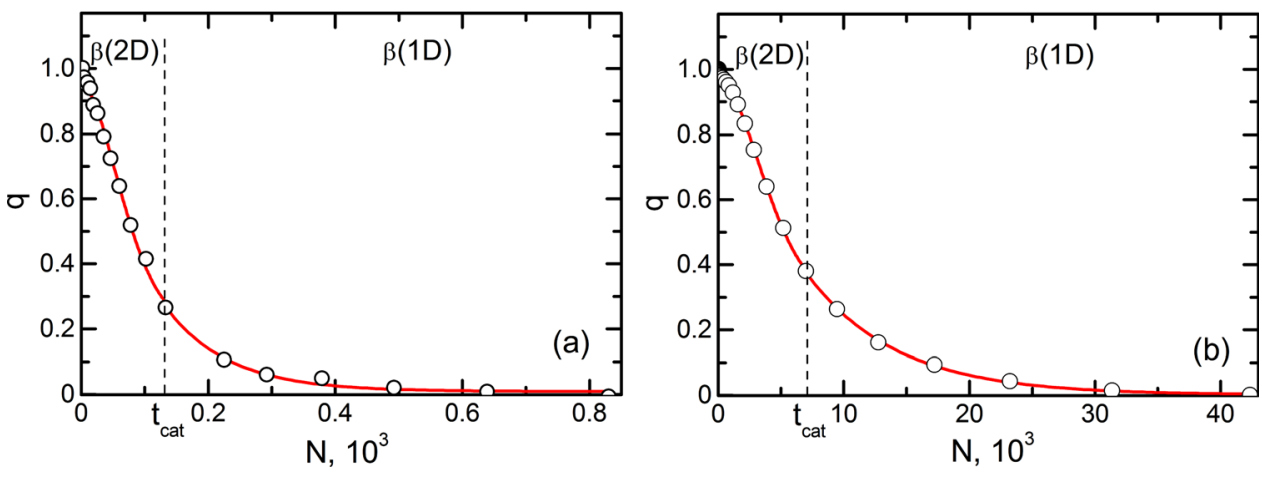

FIG. 9. The dependence of the fraction of the switched charge on the switching cycle number fitted by modified K-A formula (13) with geometrical catastrophe. Frequency of the triangular switching pulses: (a) $1 \mathrm{~Hz}$, (b) $100 \mathrm{~Hz}$. 


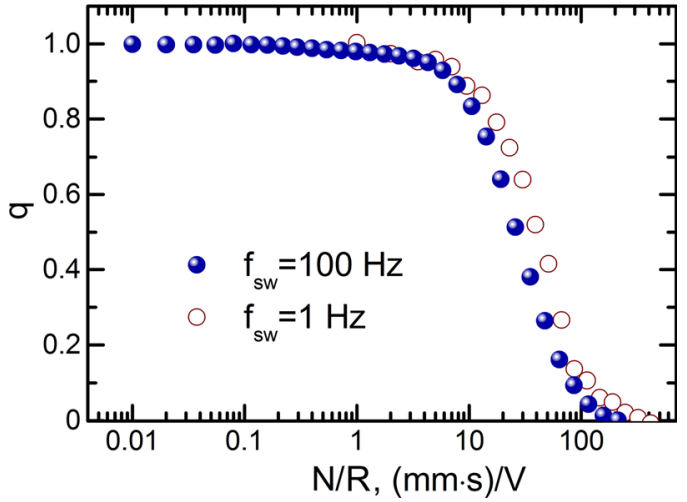

FIG. 10. Dependence of the switched charge fraction on the number of switching cycles divided by field rise rate. The amplitude of the triangular switching pulses- $500 \mathrm{~V} / \mathrm{mm}$.

when the long boundaries of CDW areas form as a result of merging of the isolated CDW areas (Fig. 7(c)). ${ }^{29}$ The interaction between the approached boundaries leads to formation of the elongated switchable areas for $q<0.4$. This fact is a clear demonstration that for switching in partially fatigued sample, the boundaries of CDW area play the role of the elongated nucleation sites. It should be noted that the catastrophe point corresponding to the fatigue degree $\gamma=0.6(q=0.4)$ is close to percolation threshold for 2D square lattice (0.593).

In the case of bipolar triangular switching pulses, Eq. (12) for the fraction of switched charge during cyclic switching can be rewritten as

$\left.q(N)=\exp \left\{-\left[(N / R) \Delta E_{C D W} / t_{0 \beta}\right]^{n}\left[1-(N / R) \Delta E_{C D W} / t_{\mathrm{m}}\right)\right]\right\}$.

Only $R$ depends on the frequency of switching pulses in this equation. Thus, the dependence of $q$ on the $N / R$ should be frequency independent for constant value of $E_{\max }$. This prediction of the model has been confirmed experimentally (Fig. 10).

It is necessary to point out that the predicted independence of the fatigue process on the frequency for cycling by rectangular pulses has been reported in several papers. ${ }^{43,44}$ The variety of the experimentally observed fatigue behaviors is caused by frequently demonstrated incomplete switching during cycling or absence of information about the used rise rate of the switching field.

\section{CONCLUSION}

We have proposed the model of the fatigue effect (decreasing of the residual polarization) during cyclic switching caused by growth of the frozen (non-switchable) domain area with charged domain walls. On the basis of the earlier experimental study of the charged domain wall growth, it was claimed that the growth of the frozen domain area in increasing field started at the sub-threshold field and changed by classical growth of domains with neutral domain walls in the fields above threshold value. The influence of the shape and frequency of the field pulses used for cycling have been considered. The uniaxial single-crystalline stoichiometric lithium tantalate produced by vapor transport equilibration with record low value of coercive field has been used as the model crystal for experimental verification of the proposed model. The formation and growth of the charged domain walls has been directly revealed by analysis of the domain images after different number of the switching cycles obtained by optical and Raman confocal microscopy. The experimentally observed fact that the fatigue degree is equal to the fraction of the frozen domain area confirmed the crucial role of the proposed fatigue mechanism. The experimentally obtained fatigue profiles have been successfully fitted by KolmogorovAvrami formula modified for finite media. The model explained the experimentally observed frequency independence of fatigue profile for rectangular pulses and frequency dependence for triangular pulses demonstrated in the paper.

\section{ACKNOWLEDGMENTS}

The research was made possible in part by RFBR (Grants 10-02-96042-r-Ural-a, 10-02-00627-a, 11-02-91066-CNRS-a, 11-02-91174-NSFC_a) and by Ministry of Education and Science (Contract 16.552 117020 ).

${ }^{1}$ D. C. Lupascu, Fatigue in Ferroelectric Ceramics and Related Issues (Springer, Berlin, 2004), p. 225.

${ }^{2}$ A. K. Tagantsev, I. Stolichnov, E. L. Colla, and N. Setter, J. Appl. Phys. 90, 1387 (2001).

${ }^{3}$ X. J. Lou, J. Appl. Phys. 105, 024101 (2009).

${ }^{4}$ P. K. Larsen, G. J. M. Dormans, D. J. Taylor, and P. J. van Veldhoven, J. Appl. Phys. 76, 2405 (1994).

${ }^{5}$ J. J. Lee, C. L. Thio, and S. B. Desu, J. Appl. Phys. 78, 5073 (1995).

${ }^{6}$ A. Bratkovsky and A. Levanyuk, Phys. Rev. Lett. 84, 3177 (2000).

${ }^{7}$ W. L. Warren, D. Dimos, B. A. Tuttle, G. E. Pike, R. W. Schwartz, P. J. Clews, and D. C. McIntyre, J. Appl. Phys. 77, 6695 (1995).

${ }^{8}$ E. L. Colla, A. K. Tagantsev, D. V. Taylor, and A. L. Kholkin, Integr. Ferroelectr. 18, 19 (1997).

${ }^{9}$ V. Ya. Shur, A. R. Akhmatkhanov, I. S. Baturin, M. S. Nebogatikov, and M. A. Dolbilov, Phys. Solid State 52, 2147 (2010).

${ }^{10}$ I. S. Baturin, A. R. Akhmatkhanov, V. Ya. Shur, M. S. Nebogatikov, M. A. Dolbilov, and E. A. Rodina, Ferroelectrics 374, 1 (2008).

${ }^{11}$ V. Ya. Shur, E. L. Rumyantsev, E. V. Nikolaeva, E. I. Shishkin, and I. S. Baturin, J. Appl. Phys. 90, 6312 (2001); V. Ya. Shur, E. L. Rumyantsev, E. V. Nikolaeva, E. I. Shishkin, and I. S. Baturin, Phys. Solid State 44, 2145 (2002).

${ }^{12}$ M. Dawber and J. F. Scott, Appl. Phys. Lett. 76, 1060 (2000).

${ }^{13}$ I. K. Yoo and S. B. Desu, Phys. Status Solidi A 133, 565 (1992).

${ }^{14}$ U. Robels, J. H. Calderwood, and G. Arlt, J. Appl. Phys. 77, 4002 (1995).

${ }^{15}$ G. Le Rhun, G. Poullain, and R. Bouregba, J. Appl. Phys. 96, 3876 (2004).

${ }^{16}$ S. B. Majumder, Y. N. Mohapatra, and D. C. Agrawal, Appl. Phys. Lett. 70, 138 (1997).

${ }^{17}$ E. L. Colla, S. Hong, D. V. Taylor, A. K. Tagantsev, N. Setter, and K. No, Appl. Phys. Lett. 72, 2763 (1998).

${ }^{18}$ A. Gruverman, O. Auciello, and H. Tokumoto, Appl. Phys. Lett. 69, 3191 (1996).

${ }^{19}$ D. S. Campbell, Philos. Mag. 7, 1157 (1962).

${ }^{20}$ A. Yu. Kudzin, T. V. Panchenko, and S. P. Yudin, Phys. Solid State 16, 1589 (1975).

${ }^{21}$ V. Ya. Shur, E. L. Rumyantsev, E. V. Nikolaeva, and E. I. Shishkin, Appl. Phys. Lett. 77, 3636 (2000).

${ }^{22}$ Y. Ishibashi and Y. Takagi, J. Phys. Soc. Jpn. 31, 506 (1971).

${ }^{23}$ V. Ya. Shur and E. L. Rumyantsev, Ferroelectrics 142, 1 (1993).

${ }^{24}$ V. Ya. Shur and E. L. Rumyantsev, Ferroelectrics 151, 171 (1994).

${ }^{25}$ A. N. Kolmogorov, Izv. Akad. Nauk SSSR, Ser. Mat. 3, 355 (1937).

${ }^{26}$ M. Avrami, J. Chem. Phys. 8, 1103 (1939).

${ }^{27}$ H. Duiker and P. Beale, Phys. Rev. B 41, 490-495 (1990).

${ }^{28}$ H. Orihara and Y. Ishibashi, J. Phys. Soc. Jpn. 61, 1919-1925 (1992).

${ }^{29}$ V. Shur, E. Rumyantsev, and S. Makarov, J. Appl. Phys. 84, 445 (1998).

${ }^{30}$ R. Abe, J. Phys. Soc. Jpn. 14, 633 (1959).

${ }^{31}$ V. Ya. Shur and E. L. Rumyantsev, Ferroelectrics 191, 319 (1997). 
${ }^{32}$ E. Soergel, Appl. Phys. B 81, 729 (2005).

${ }^{33}$ L. Tian, V. Gopalan, and L. Galambos, Appl. Phys. Lett. 85, 4445 (2004).

${ }^{34}$ D. S. Hum, R. K. Route, G. D. Miller, V. Kondilenko, A. Alexandrovski, J. Huang, K. Urbanek, R. L. Byer, and M. M. Fejer, J. Appl. Phys. 101, 93108 (2007).

${ }^{35}$ V. Ya. Shur, A. R. Akhmatkhanov, I. S. Baturin, and E. V. Shishkina, J. Appl. Phys. 111, 014101 (2012).

${ }^{36}$ R. L. Byer, J. Nonlinear Opt. Phys. Mater. 6, 549 (1997).

${ }^{37}$ P. F. Bordui, R. G. Norwood, D. H. Jundt, and M. M. Fejer, J. Appl. Phys. 71, 875 (1992)

${ }^{38}$ P. S. Zelenovskiy, V. Ya. Shur, P. Bourson, M. D. Fontana, D. K. Kuznetsov, and E. A. Mingaliev, Ferroelectrics 398, 34 (2010).
${ }^{39}$ V. Ya. Shur, P. S. Zelenovskiy, M. S. Nebogatikov, D. O. Alikin, M. F. Sarmanova, A. V. Ievlev, E. A. Mingaliev, and D. K. Kuznetsov, J. Appl. Phys. 110, 052013 (2011).

${ }^{40}$ F. Jona and G. Shirane, Ferroelectric Crystals (Pergamon, Oxford, 1962), p. 402.

${ }^{41}$ J. C. Barfoot and G. W. Taylor, Polar Dielectrics and Their Applications (MacMillan, London, 1979), p. 465.

${ }^{42}$ I. S. Baturin, M. V. Konev, A. R. Akhmatkhanov, A. I. Lobov, and V. Ya. Shur, Ferroelectrics 374, 136 (2008).

${ }^{43}$ M. Grossmann, D. Bolten, O. Lohse, U. Boettger, R. Waser, and S. Tiedke, Appl. Phys. Lett. 77, 1894 (2000).

${ }^{44}$ B. G. Chae, C. H. Park, Y. S. Yang, and M. S. Jang, Appl. Phys. Lett. 75, 2135 (1999). 